\title{
“Handwriting \& Individuality”: A Psychological, Biomechanical and Growth \& Developmental Perspective of Handwriting
}

\author{
Aggarwal Sapna ${ }^{1}$
}

\section{ABSTRACT}

Whenever we think of handwriting assessment all that comes to mind is the non-empirical method of Handwriting Analysis or Graphology. We have never seriously considered handwriting as an independent entity. In this article I have tried presenting few aspects with which we can try and understand handwriting i.e. psychological, biomechanical and growth \& developmental. From decades researchers have tried establishing empirical grounds for Graphology. But its time we accept that graphology has no scientific basis and is a pseudoscience which claims to assess things that even the scientific world is hesitant to affirm.

Keywords: Handwriting, Graphology.

Personality psychologists and researchers have time and again tried to come up with tools to assess the dynamism of human personality, such as Self Report Inventories, Performance Based Measures, Observations, Interviews, etc. But if we evaluate the administration of these so-called standard tools in meticulously, we will find that in one way or the other they lack the ability to tap into the reality of the most complex machine on earth - the human mind. Amongst these clinical methods of personality assessment many interesting techniques have also come into existence. These techniques are neither considered a complete science nor a complete art and are termed as pseudoscience. One such method of personality assessment is handwriting analysis.

Annette Poizner, MSSW, Ed.D. explains that, Handwriting Analysis which is more popularly known as "Graphology" engages the practitioner in analyzing the structural graphic elements of a writer's handwriting, in order to derive information about the writer's personality. With the aid of graphological theory, graphologists identify the qualities, traits, attitudes, sentiments or postures that seem indicated in the handwriting; they further seek insight into how these aspects of selfhood may integrate together to constitute the dynamic organization that we recognize as the "personality" of that writer.

Handwriting analysis claims to assess things which even the scientific world is hesitant to affirm. Further, those who believe in graphology as science assume that when performing an analysis,

\footnotetext{
${ }^{1}$ Bangalore, India

*Corresponding Author

(c) 2015 I A Sapna; licensee IJIP. This is an Open Access Research distributed under the terms of the Creative Commons Attribution License (http://creativecommons.org/licenses/by/2.0), which permits unrestricted use, distribution, and reproduction in any Medium, provided the original work is properly cited.
} 


\section{"Handwriting \& Individuality": A Psychological, Biomechanical and Growth \& Developmental Perspective of Handwriting}

graphologists examine all facets of the writing's letter forms and spatial arrangements, while also attending to the quality of the writing's ink trail or ductus.

Handwriting Analysis as an empirical method of personality assessment is in controversy from decades. Some researchers say it is a potential method to assess personality and on the other hand some researchers say it's not. E. A. Rundquist says that, many scientists try distinguishing between the well-established branch of "forensic graphology" devoted to problems of personal identification and the branch devoted to character analysis; he stresses the need for detailed research studies; he have recognized many of the pitfalls that need be avoided in carrying out such studies.

Handwriting analysis definitely has serious methodological deficits. These shortcomings are not justifiable in the empirical research world and need in-depth research aiming at testing the methodology of this tool.

Tough handwriting analysis is not a valid tool to assess personality, but its failure as a science cannot hamper the truth that "handwriting as an independent entity" has a lot to promise. Researchers need to forgo the old methods attached to assessment of handwriting and try researching it with a new vision and a new perspective.

\section{Understanding Handwriting as an Independent Entity}

Diana Harrison say that, handwriting is a complex motor skill that is the combination of sensory, neurological, and physiological impulses. Factors such as visual perception and acuity, comprehension of form, central nervous system pathways, and the anatomy and physiology of the bones and muscles of the hand and arm all combine to produce the desired output.

Handwriting is not limited to any one functional aspect. Hence, in order to better understand it we can try exploring its psychological, biomechanical and growth and developmental aspect.

\section{Psychological Aspect of Handwriting}

Diana Harrison suggests that, most people learn to write by copying letter formations from a copybook at a young age. The ability to reproduce the letter formations varies from one person to the next and is based on each writer's perception of the image and his or her ability (motor skills) to reproduce that visual perception. The act of handwriting is mastered through practice and repetition. Once this occurs, writers focus on the subject matter rather than the physical act of writing and deviate from the copybook forms, interjecting their own individual characteristics. The writing becomes a pattern of subconscious, habitual formations that are repeated from one writing to the next.

Just as personality is dynamic and keeps changing, if we observe handwriting of an individual we will find that the way one writes also changes from time to time, situation to situation and day to day. Sometimes, what we write or how we sign on a particular day also has so much 


\section{"Handwriting \& Individuality": A Psychological, Biomechanical and Growth \& Developmental Perspective of Handwriting}

variation to offer. This definitely tells something, it may be the relationship of handwriting and personality, handwriting and behavior or may be handwriting and moods.

\section{Biomechanical Aspect of Handwriting}

If handwriting is a movement and it is controlled by the faculties of brain, then it should be analyzed the way running, walking, jumping etc. are analyzed and biomechanists can try answering that - "why there are differences in handwriting of each and every individual”.

If we observe handwriting as a movement then we will find that as there are differences in gait (walking style) of every individual, similarly there are differences in the handwritings. Here, if we try to assume that gait of one individual is better than the other individual because they have personality differences, we might not be correct. But it won't be incorrect to assume that one gait is better/worse than other in terms of mechanical efficiency. Similarly, it will not be logical to assume that differences in handwriting (as a movement) are representative of individual personalities but it won't be incorrect to say that one handwriting looks better/worse than other in terms of spacing, legibility, margins, letter size etc.

\section{Handwriting as an Aspect of Growth and Development}

If changes and variations in handwriting are triggered by the process of growth and development than it won't be illogical to assume that handwriting changes, as an individual grows old and becomes mentally and physically mature. But this change has to support the assumption that at childhood brain is developing and so are the movements. These skills start to develop and become stronger and stronger as an individual matures and ultimately during old age, where we once again begin to loose control over movements and coordination, the handwriting skills again begin to deteriorate.

\section{CONCLUSION}

As we talk about handwriting assessment all we can think of is Handwriting Analysis, a method which is not empirical and claims of things which even the psychologists hesitate to state. But this should not hamper the possibilities "handwriting as an independent entity" has to offer. There is observable dynamism in handwriting of each and every individual and it need to be researched. Many attempts have been made to validate the method of handwriting analysis and it won't be illogical to say that it's better if we try understanding aspects of an individual's handwriting with new perspectives and conclude that handwriting analysis is no more than a pseudooscience. 


\section{REFERENCES}

Diana Harrison, Ted M. Burkes and Danielle P. Seiger, Handwriting Examination: Meeting the Challenges of Science and the Law. Forensic Science Communications. October 2009 Volume - Number 4. Web 26 Oct 2013, https://www2.fbi.gov/hq/lab/fsc/backissu/oct2009/review/2009_10_review02.htm

Poizner Annette, MSSW, Ed.D. Graphology in Clinical Practice. Psychologica, Volume 24, Number 1, Spring 2004. Web. 26 Oct 2013.

http://www.annettepoizner.com/psychvol24-1.pdf

Rundquist, E.A. The Assessment of Graphology. Psychology Journal Article Collection. Web. 26 Oct 2013. < http://www.all-about-psychology.com/handwriting-analysis.html 